\title{
Propriedades de pasta de mesclas de farinha integral com farinha refinada usadas na produção de pães
}

\author{
Pasting properties of whole and refined wheat flour blends used for bread production \\ Tatiana Oro $^{\mathrm{I}^{*}}$ Valéria Maria Limberger $^{\mathrm{I}}$ Martha Zavariz de Miranda $^{\mathrm{II}}$ \\ Neila Silvia Pereira dos Santos Richards ${ }^{\text {III }}$ Luiz Carlos Gutkoski ${ }^{\mathrm{IV}}$ Alicia de Francisco ${ }^{\mathrm{I}}$
}

\section{RESUMO}

Na produção de pães integrais de trigo no Brasil, são utilizadas mesclas de farinha integral (FI) com farinha refinada (FR), as quais diferem em sua composição. Assim, o objetivo deste estudo foi investigar as propriedades de pasta de mesclas de FI com FR. Foram analisadas amostras com 100\% de FI, 100\% de $F R$ (controles) e mesclas de FI com FR nas proporções de 20, 40, 60 e 80\%, em equipamento RVA (Rapid Visco Analyzer). A temperatura de pasta das amostras foi semelhante. A viscosidade máxima diminuiu proporcionalmente com o aumento de FI na mescla. Maiores valores de quebra e de viscosidade mínima à temperatura constante foram encontrados em farinhas com pico de viscosidade elevado. Os valores de viscosidade final e de tendência à retrogradação diminuíram à medida que aumentou o teor de FI na mescla. As propriedades de pasta das mesclas de FI com FR devem ser vistas de forma conjunta, pois afetam a produção de pão e o armazenamento. Os resultados mostraram também que o RVA pode ser usado para ajudar a formular mesclas de FI/FR com certas propriedades de pasta.

Palavras-chave: trigo moído, processamento de pão, características de viscosidade, $R V A$.

\section{ABSTRACT}

In whole wheat bread production in Brazil blends of whole flour (WF) with refined flour (RF) are used, which differ in their composition. Thus, the objective of this study was to investigate the pasting properties of WF with RF blends. Samples with 100\% of WF, $100 \%$ of RF (controls) and blends of WF with $R F$ in the proportions of 20,40,60 and $80 \%$ were analyzed, in a RVA (Rapid Visco Analyzer) equipment. The samples pasting temperatures were similar. The peak viscosity decreased proportionally with the increase of WF in blends. Higher values of breakdown and holding strength were found in flour with high viscosity peak. Final viscosity and setback values decreased as the content of $W F$ in blends increased. The pasting properties of WF with RF blends must be seen in a combined way, because they affect bread production and storage. The results also showed that RVA can be used to help in formulating WF/RF blends with certain pasting properties.

Key words: wheat meal, bread process, viscosity characteristics, RVA.

\section{INTRODUÇÃO}

A produção mundial de trigo (Triticum aestivum) chega a ultrapassar 680 milhões de toneladas ao ano (IGC, 2011). No Brasil, a produção anual oscila entre 5 e 6 milhões de toneladas e o consumo anual é da ordem de 10 milhões de toneladas. $\mathrm{O}$ consumo de trigo tem aumentado na ordem de 2 a $5 \%$ ao ano. Paralelamente, as importações de trigo têm aumentado, já que sua produção interna não atende à demanda de consumo (CONAB, 2011; COSTA, 2008).

Cerca de $75 \%$ do trigo consumido no país é destinado à produção de farinha refinada e cerca de $5 \%$ à produção de farinha integral (FIEP, 2006), cujo consumo vem crescendo devido a apelos de saúde dos produtos integrais, principalmente por serem ricos em fibras.

'Universidade Federal de Santa Catarina (UFSC), 88040-970, Florianópolis, SC, Brasil. E-mail: tatianaoro@ hotmail.com. *Autor para correspondência.

"Embrapa Trigo, Passo Fundo, RS, Brasil.

IIIUniversidade Federal de Santa Maria (UFSM), Santa Maria, RS, Brasil.

${ }^{\text {IV }}$ Fundação Universidade de Passo Fundo (UPF), Passo Fundo, RS, Brasil. 
O pão é um dos alimentos mais consumidos e componente básico na alimentação dos brasileiros, sendo o pão francês o mais consumido (58\% da produção de pães), seguido do pão de forma (ABIP, 2011). Entretanto, o mercado de pães especiais, que agregam grãos integrais, cereais, ervas e outros ingredientes com apelo nutricional, apresentam crescimento estimado de $15 \%$ para os próximos cinco anos, contrastando com os $10 \%$ previstos para o restante do setor de panificação (PROPAN, 2011).

As farinhas integrais consistem de grãos moídos em sua totalidade e são constituídas pelo farelo, pelo gérmen e pelo endosperma (WHOLE GRAINS COUNCIL, 2011), possuindo granulometria homogênea. No Brasil, como não há legislação específica que garanta que o trigo seja moído integralmente, também pode ser considerada farinha integral de trigo aquela com adição de farelo à farinha refinada. Embora seja uma facilidade para o moinho usar o farelo, que é um subproduto da moagem, este possui maior granulometria, deixando a farinha integral desuniforme, e que não deveria ser considerada como tal.

$\mathrm{O}$ amido é o componente mais relevante das farinhas (COPELAND et al., 2009), representando 60 a $70 \%$ do peso seco do grão e 65 a $75 \%$ dos componentes da farinha refinada (SHEWRY, 2009), atuando como ingrediente funcional na indústria de alimentos.

$\mathrm{O}$ processamento do amido geralmente envolve aquecimento na presença de água sob cisalhamento e posterior resfriamento. Durante o aquecimento, os grânulos de amido são gelatinizados e, no resfriamento, as macromoléculas constituintes dos grânulos de amido retrogradam. Entender as etapas que ocorrem durante a gelatinização e a retrogradação são passos necessários para melhor predizer as propriedades funcionais do amido processado (COPELAND et al., 2009). Isso ocorre na panificação, durante a etapa de produção ou processamento e depois no armazenamento.

Não existem métodos analíticos oficiais para a avaliação da qualidade tecnológica de farinha integral de trigo e pouco se conhece sobre o comportamento de viscosidade do amido, presente nas mesclas usadas para produção de pães contendo farinha integral de trigo. Essa falta se dá pela carência de pesquisas científicas que caracterizem melhor este tipo de produto. Entre os equipamentos usados para avaliar as propriedades de pasta do amido, há o analisador rápido de viscosidade (RVA), o viscoamilógrafo (Brabender) e o Mixolab (Chopin).

$\mathrm{Na}$ produção de pães integrais no Brasil, em geral, são utilizadas misturas com teores máximos de $60 \%$ de farinha integral com farinha refinada, ajustando-se à preferência do consumidor, que são pães mais crescidos e macios diferentes daqueles produzidos apenas com farinha integral. Dessa forma, o objetivo deste trabalho foi investigar as propriedades de pasta de mesclas de farinha de trigo integral com farinha refinada, usadas na produção de pães no Brasil.

\section{MATERIAL E MÉTODOS}

Foram obtidas amostras de grãos de trigo (Triticum aestivum L.), safra de 2009, e farinha integral de trigo a partir de dois diferentes fornecedores comerciais brasileiros, que foram denominados (A) e (B). Os grãos foram processados para a obtenção de farinha de trigo refinada em moinho experimental de laboratório Brabender, modelo Quadrumat Senior (BRABENDER OHG, Alemanha). Após o processo de moagem, foram preparadas misturas contendo diferentes proporções de Farinha Integral (FI) e Farinha Refinada (FR), que foram codificadas como: FR, com $100 \%$ de FR; 20FI-80FR, mescla com $20 \%$ de FI e $80 \%$ de FR; 40FI-60FR, mescla com $40 \%$ de FI e $60 \%$ de FR; 60FI-40FR, mescla com $60 \%$ de FI e $40 \%$ de FR; 80 FI-20FR, mescla com $80 \%$ de FI e $20 \%$ de FR; e FI, com $100 \%$ de FI.

De acordo com a legislação brasileira (BRASIL, 2010), em relação às amostras utilizadas neste estudo, a FR (A) é classificada como originada de trigo Classe Melhorador e a FR (B), como originada de trigo Classe Pão. Essas amostras apresentam tamanho de partícula dentro dos limites estabelecidos pela legislação brasileira (BRASIL, 2005), em que mais de $95 \%$ da amostra testada é capaz de passar através de um tamis de $250 \mu \mathrm{m}$ de abertura (dados não publicados). Para as FI e mesclas de FI e FR, não existe legislação específica no Brasil.

As propriedades de pasta das FR, das FI e das misturas FI-FR foram avaliadas em analisador rápido de viscosidade (RVA), modelo RVA-3D, equipado com software Termocline for Windows, versão 3.1, de acordo com o método 76-21 da AACC (2000). O perfil de viscosidade das amostras foi analisado na base de $12,3 \% \mathrm{p} / \mathrm{p}$ (3,5g de farinha e $25 \pm 0,1 \mathrm{~mL}$ de água destilada), corrigidas para $14 \%$ de umidade. O perfil de temperatura utilizado foi o Standard 1 (amostras mantidas a $50^{\circ} \mathrm{C}$ por 1 minuto, aquecidas até $95^{\circ} \mathrm{C}$ em 4:45 minutos e mantidas nessa temperatura durante 7:15 minutos, resfriadas a $50^{\circ} \mathrm{C}$ até o tempo de 11 minutos e mantidas nessa temperatura até o total de 13 minutos de análise), sendo considerados os parâmetros: temperatura de 
pasta, viscosidade máxima, viscosidade mínima à temperatura constante, que é ligada à quebra e viscosidade final, associada à tendência à retrogradação. Na figura 1, pode-se observar uma curva de viscosidade típica do RVA.

A análise estatística dos resultados foi realizada com o programa SAS for Windows, sendo os dados experimentais submetidos à análise de variância (ANOVA) e as médias comparadas pelo teste de Tukey, em nível de 5\% de probabilidade.

\section{RESULTADOS E DISCUSSÃO}

Os resultados do comportamento de pasta dos amidos das amostras de farinha A e B e suas misturas estão apresentados na tabela 1 e ilustrados na figura 2 ( $a$ e b).

Quando o amido é aquecido em excesso de água, seus grânulos intumescem e a organização cristalina decompõe-se para formar regiões amorfas. Essa desordem molecular é denominada gelatinização (KOHYAMA et al., 2004) e é definida pelo aumento inicial rápido da viscosidade, que ocorre entre os 3-5 minutos iniciais (ZENG et al., 1997).

Além de apontar a temperatura mínima para que uma amostra seja cozida, a temperatura de pasta indica a temperatura na qual a viscosidade começa a aumentar durante o período de aquecimento (SINGH et al., 2011).
Para as amostras dos fornecedores A e $\mathrm{B}$, foram obtidas temperaturas de pasta de $50,2^{\circ} \mathrm{C}$ para as amostras FI (A) e $80 \mathrm{FI}-20 \mathrm{FR}$ (B) e $53,87^{\circ} \mathrm{C}$ para a amostra FI (B), as quais não diferiram entre si $(\mathrm{P} \leq 0,05)$, sugerindo que as amostras estudadas são semelhantes quanto às características de gelatinização.

De acordo com JANE et al. (1999), a maior parte dos amidos contém entre 20 e $30 \%$ de amilose e entre 70 e $80 \%$ de amilopectina, razão que varia de acordo com a fonte botânica. Amidos que consistem em grande parte de amilopectina com elevadas proporções de cadeias ramificadas podem apresentar temperatura de gelatinização elevada (FRANCO et al., 2002). Isso pode explicar o comportamento das farinhas deste estudo, que apresentaram temperaturas de pasta menores que as temperaturas de $95^{\circ} \mathrm{C}$ e $82,2^{\circ} \mathrm{C}$, relatadas por RAGAEE \& ABDEL-AAL (2006) e LEON et al. (2010), respectivamente. Segundo RAGAEE \& ABDEL-AAL (2006), farinhas refinadas podem apresentar maior temperatura de pasta que as farinhas integrais, por possuírem maior teor de amido na mesma porção, porém isto não pode ser observado nos resultados do presente estudo.

A viscosidade máxima é um parâmetro relacionado à capacidade do amido em absorver água, e, consequentemente ao grau de intumescimento dos grânulos de amido durante o aquecimento. Assim, quando existem grandes quantidades de grânulos com elevada capacidade de intumescimento, ocorrem elevados picos de viscosidade.

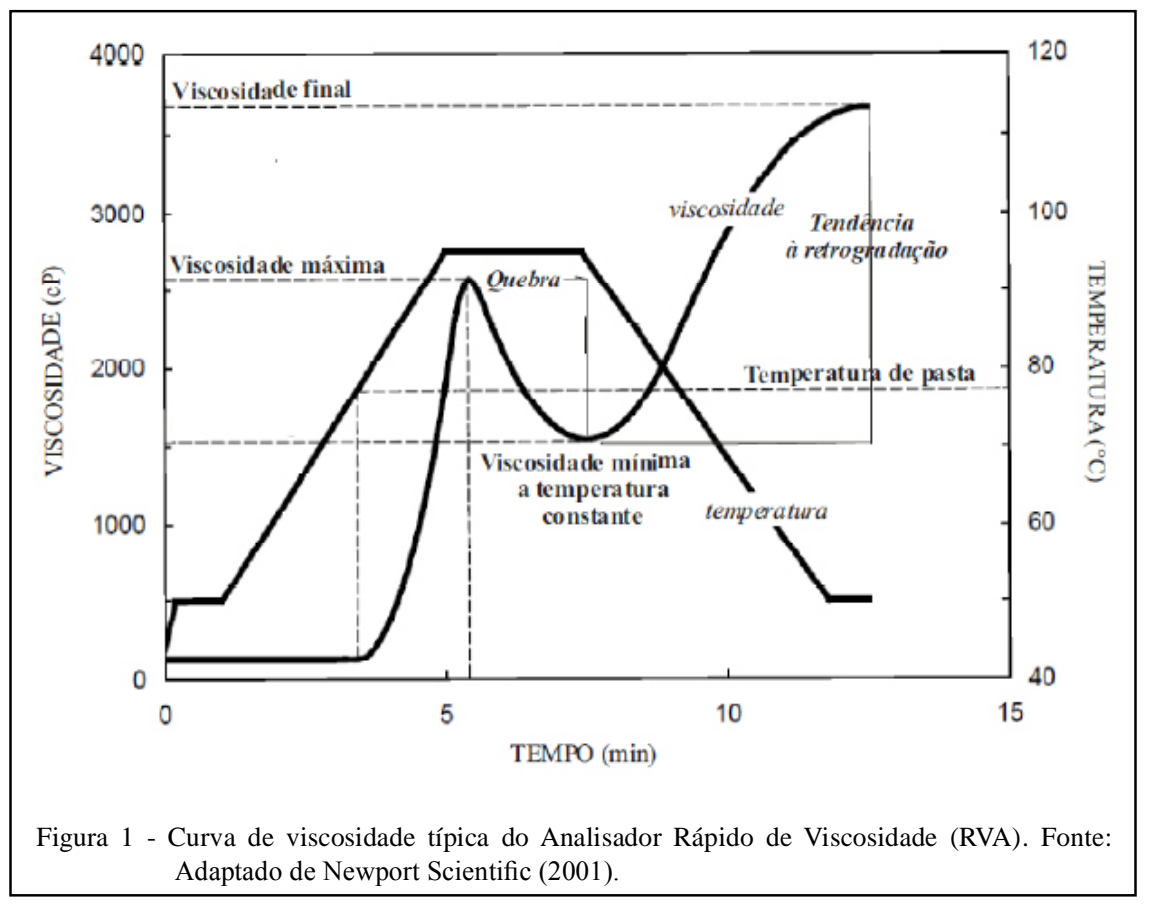

Ciência Rural, v.43, n.4, abr 2013. 
Tabela 1 - Propriedades de pasta das farinhas das amostras de trigo A e B e suas mesclas.

\begin{tabular}{|c|c|c|c|c|c|c|}
\hline Amostra* & Temp Pasta $\left({ }^{\circ} \mathrm{C}\right)$ & Visc Máx (cP) & $\operatorname{Visc} \operatorname{Min}(C p)$ & Quebra (cP) & Vise final $(\mathrm{cP})$ & Tend Retrog $(\mathrm{cP})$ \\
\hline FR (A) & $52,08^{\mathrm{a}}+1,94$ & $2032,0^{\mathrm{d}}+11,31$ & $1733,5^{\mathrm{cd}}+10,6$ & $298,5^{\mathrm{e}}+0,71$ & $2682,5^{\mathrm{e}}+6,36$ & $949,0^{\mathrm{f}}+16,97$ \\
\hline 20FI-80FR (A) & $52,40^{\mathrm{a}}+3,18$ & $1807,0^{\mathrm{ef}}+15,56$ & $1482.0^{\mathrm{e}}+9,9$ & $325,0^{\mathrm{ed}}+5,66$ & $2532,5^{\mathrm{f}}+41,72$ & $1050,5^{\mathrm{ef}}+31,82$ \\
\hline $40 \mathrm{FI}-60 \mathrm{FR}(\mathrm{A})$ & $52,97^{\mathrm{a}}+0,74$ & $1604,0^{\mathrm{g}}+31,11$ & $1317,5^{\mathrm{f}}+24,7$ & $286,5^{\mathrm{ef}}+6,36$ & $2396,0^{\mathrm{g}}+2,83$ & $1078,5^{\mathrm{efd}}+27,58$ \\
\hline $60 \mathrm{FI}-40 \mathrm{FR}(\mathrm{A})$ & $50,67^{\mathrm{a}}+0,81$ & $1432,5^{\mathrm{h}}+9,19$ & $1168,0^{\mathrm{gf}}+5,6$ & $264,5^{\mathrm{ef}}+3,54$ & $2348,5^{9}+21,92$ & $1180,5^{\text {cehd }}+27,58$ \\
\hline $80 \mathrm{FI}-20 \mathrm{FR}(\mathrm{A})$ & $51,05^{\mathrm{a}} \pm 0,57$ & $1218,5^{\mathrm{i}} \pm 17,68$ & $1025,5^{\mathrm{g}} \pm 23,3$ & $193,0^{f} \pm 5,66$ & $2132,0^{\mathrm{h}} \pm 14,14$ & $1106,5^{\mathrm{ced}} \pm 37,48$ \\
\hline FI (A) & $50,20^{\mathrm{a}}+0,14$ & $1891,5^{\mathrm{e}} \pm 45,96$ & $1549,5^{\mathrm{e}}+53,0$ & $342,5^{\mathrm{ed}}+7,07$ & $3203,5^{\mathrm{b}}+44,55$ & $1654,0^{\mathrm{a}}+8,49$ \\
\hline FR (B) & $51,30^{\mathrm{a}}+1,34$ & $2733,5^{\mathrm{a}} \pm 7,78$ & $2093.5^{\mathrm{a}}+19,1$ & $640,0^{\mathrm{b}}+26,87$ & $3162,5^{\mathrm{b}}+20,51$ & $1069,0^{\text {ef }}+39,60$ \\
\hline $20 \mathrm{FI}-80 \mathrm{FR}(\mathrm{B})$ & $50,67^{\mathrm{a}} \pm 0,04$ & $2527,0^{\mathrm{b}}+0,00$ & $1869.0^{\mathrm{cb}}+45,2$ & $658,0^{\text {ha }} \pm 45,25$ & $3000,5^{c}+10,61$ & $1131,5^{\text {cehd }}+34,65$ \\
\hline $40 \mathrm{FI}-60 \mathrm{FR}(\mathrm{B})$ & $51,20^{\mathrm{a}} \pm 1,20$ & $2263,0^{c}+8,49$ & $1615.0^{\mathrm{ed}}+56,5$ & $648,0^{\mathrm{b}}+65,05$ & $2866,0^{d}+16,97$ & $1251,0^{\mathrm{b}}+39,60$ \\
\hline $60 \mathrm{FI}-40 \mathrm{FR}(\mathrm{B})$ & $50,72^{\mathrm{a}}+0,74$ & $2054,5^{d}+4,95$ & $1518.5^{e}+17,6$ & $536,0^{c}+12,73$ & $2736,5^{\mathrm{e}}+23,33$ & $1218,0^{\mathrm{cbd}}+41,01$ \\
\hline $80 \mathrm{FI}-20 \mathrm{FR}(\mathrm{B})$ & $50,20^{\mathrm{a}} \pm 0,00$ & $1729,5^{\mathrm{f}} \pm 23,33$ & $1305.0^{\mathrm{f}} \pm 31,1$ & $424,5^{\mathrm{d}} \pm 7,78$ & $2539,5^{\mathrm{f}} \pm 60,10$ & $1234,5^{\mathrm{cb}} \pm 28,99$ \\
\hline $\mathrm{FI}(\mathrm{B})$ & $53,87^{\mathrm{a}}+4,91$ & $2683,0^{\mathrm{a}}+55,15$ & $1929.5^{\mathrm{b}}+77,1$ & $753,5^{\mathrm{a}}+21,92$ & $3565,0^{\mathrm{a}}+8,49$ & $1635,5^{\mathrm{a}}+68,59$ \\
\hline
\end{tabular}

Valores na mesma coluna, seguidos por letras diferentes, diferem significativamente entre si $(\mathrm{P}=0,05)$. Resultados expressos como média de duas determinações \pm desvio padrão. Temp Pasta: temperatura de pasta; Visc Máx: viscosidade máxima; Visc Mín: viscosidade mínima à temperatura constante; Quebra: viscosidade máxima menos viscosidade mínima à temperatura constante; Visc Final: viscosidade final; Tend Retrog: tendência a retrogradação: viscosidade final menos viscosidade mínima à temperatura constante. cP: centipoise. FR: farinha refinada. FI: farinha integral. (A) e (B): fornecedores das amostras. *Proporção entre FI e FR.

Foi observada diminuição gradual nos valores de viscosidade máxima das amostras $(\mathrm{P} \leq 0,05)$, de acordo com o aumento do teor de farinha integral na mistura, sendo obtidos 2032 e 2733,5 cP para FR e 1218,5 e 1729,5 cP para as amostras 80FI-20FR dos fornecedores A e B, respectivamente. Os valores obtidos para as amostras de $100 \%$ de FI foram os mais próximos aos das amostras de $100 \%$ de FR, 1891,5 e 2683 cP para FI dos fornecedores A e B, respectivamente. Isso possivelmente decorre das interações químicas entre amido e fibras, proteínas, lipídios, presentes na FI, que afetam o comportamento de viscosidade das farinhas.

De acordo com RAGAEE \& ABDELAAL (2006), a viscosidade máxima mais elevada nas FR's pode ser atribuída, em parte, ao seu maior teor de amido e também a diferenças no teor de proteínas entre a FR e as FI. SINGH et al. (2011) afirmaram que a absorção de água pelos grânulos de amido pode ser inibida na presença de proteínas, o que poderia acarretar menores valores para viscosidade máxima nas FI e suas misturas, visto que elas possuem maior teor proteico que as FR. BRANDOLINI et al. (2010) encontraram valores de $1510 \mathrm{cP}$ e $1420 \mathrm{cP}$ para farinhas de trigo integral e refinada, respectivamente.

Segundo TESTER \& MORRISON (1990), as propriedades de gelatinização e de poder de intumescimento dos grânulos são determinadas primeiramente pela amilopectina. A amilose atuaria parcialmente como diluente da amilopectina e parcialmente como um inibidor do intumescimento, especialmente quando complexada com lipídios. De acordo com JANE et al. (1999), a amilopectina contribui para o intumescimento dos grânulos e propriedades de pasta, enquanto que a amilose e os lipídios inibem este intumescimento. Além disso, de acordo com os autores, o comprimento das cadeias de amilopectina e o tamanho da molécula de amilose produzem efeitos sinérgicos na viscosidade de pasta do amido.

A quebra é um fenômeno associado à estabilidade dos grânulos de amido ao aquecimento, pois, durante esse período, os grânulos de amido rompem-se, a amilose lixivia do interior dos grânulos e contribui para a viscosidade da fase contínua em dispersões amido-água e na taxa de retrogradação durante o resfriamento (LEON et al., 2010; ZENG et al., 1997). De acordo com ZENG et al. (1997), menores teores de amilose são correlacionados com alto pico de viscosidade de pasta, maior quebra e menor viscosidade final do amido de trigo. Segundo SINGH et al. (2011), baixos valores de quebra indicam melhor resistência ao cisalhamento.

Para este parâmetro, foi percebida a mesma tendência observada para viscosidade máxima, em que os valores obtidos diminuíram de acordo com o aumento da proporção de FI na mistura. Foram obtidos 298,5 e 640,0 cP para FR e 193,0 e 424,5 cP para as amostras $80 \mathrm{FI}-20 \mathrm{FR}$ dos fornecedores A e B, respectivamente. 

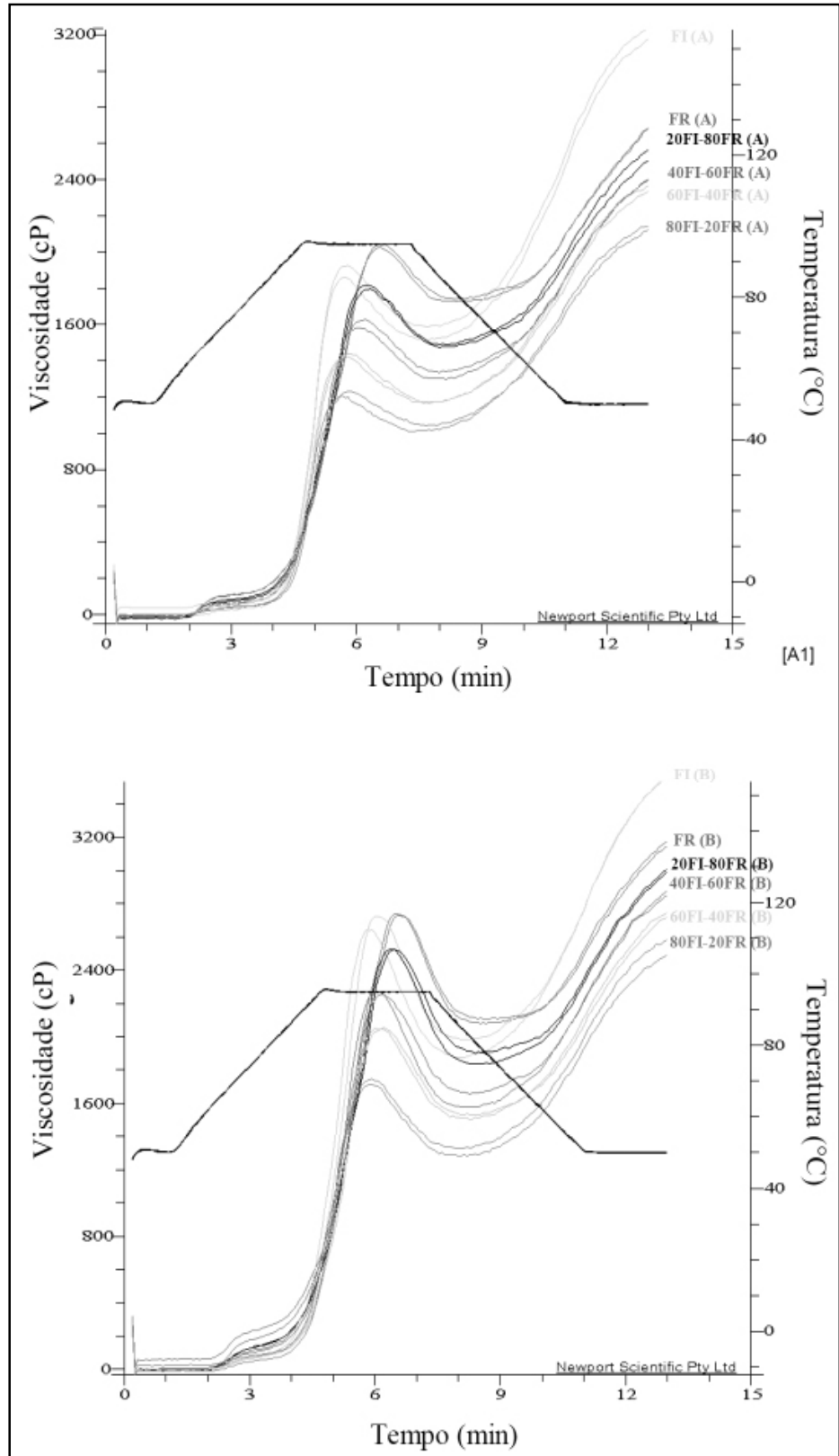

Figura 2 - Propriedades de pasta das amostras dos fornecedores A (2a) e B (2b), analisadas em RVA, em que: FR: farinha refinada; FI: farinha integral e FI-FR: Proporção entre FI e FR.

Os valores obtidos entre as amostras dos dois fornecedores para as farinhas e suas respectivas misturas diferiram significativamente entre si $(\mathrm{P} \leq 0,05)$, sendo que, para as FI dos fornecedores Ae B, foram obtidos valores de quebra de 342,5 e $753,5 \mathrm{cP}$. Isso pode ser explicado pelo fato de que farinhas com pico de viscosidade mais elevado produzem maiores valores de quebra, pois seus grânulos de amido apresentam maior capacidade de intumescimento
(RAGAEE \& ABDEL-AAL, 2006). Possivelmente, farinhas que apresentam esse comportamento também deverão possuir elevado teor de amilopectina, que é a responsável pelas propriedades de intumescimento e gelatinização. Provavelmente, essa afirmação justifique o ocorrido com as amostras do fornecedor $\mathrm{B}$, que apresentaram pico de viscosidade elevado e maior valor de quebra, assim como ocorreu no estudo realizado por RAGAEE \& ABDEL AAL (2006), em 
amostras de farinha de trigo soft, farinha integral de cevada e farinha de trigo duro.

A viscosidade mínima à temperatura constante é configurada pelo valor da viscosidade no momento em que a temperatura é mantida a $95^{\circ} \mathrm{C}$ e que juntamente com a quebra, que é calculada pela diferença entre o valor da viscosidade máxima e da viscosidade mínima à temperatura constante, está associada à estabilidade dos grânulos de amido em relação ao aquecimento.

Os resultados obtidos para viscosidade mínima à temperatura constante, assim como os obtidos para viscosidade máxima e quebra, apresentaram valores que diminuíram à medida que aumentou o teor de FI nas misturas. Para as amostras do fornecedor $\mathrm{A}$, foram obtidos valores entre 1025,5 e 1733,5 cP (amostras 80FI-20FR (A) e FR (A), $\mathrm{P} \leq 0,05$ ) e, para as amostras 80FI-20FR (B) e FR (B), do fornecedor B, valores entre 1305 e 2093,5 cP, respectivamente.

A viscosidade final representa o valor da viscosidade após a amostra ser resfriada a $50^{\circ} \mathrm{C}$ e mantida nesta temperatura. A viscosidade final, associada à tendência à retrogradação, que é calculada pela diferença entre a viscosidade final e a viscosidade mínima à temperatura constante, representam uma medida da tendência do amido em retrogradar.

Para a viscosidade final, foram obtidos valores entre 2682,5 e 3162,5 cP para FR e 2132 e 2539 cP para as amostras 80FI-20FR dos fornecedores A e $\mathrm{B}$, respectivamente (que diferiram significativamente, $\mathrm{P} \leq 0,05)$. As FI apresentaram valores de 3203,5 e $3565 \mathrm{cP}$, para as amostras dos fornecedores A e $\mathrm{B}$, respectivamente, deixando evidente que as amostras dos dois fornecedores apresentaram a mesma tendência e que, possivelmente, refletem o comportamento real das farinhas integrais existentes no mercado brasileiro.

Durante o resfriamento da amostra, a reassociação entre as moléculas constituintes do amido, especialmente as de amilose, resultam na formação de uma estrutura de gel e a viscosidade aumenta até atingir a viscosidade final. Essa fase é descrita como região de tendência à retrogradação e está relacionada com reorganização das moléculas de amido (RAGAEE \& ABDEL-AAL, 2006). O valor da tendência à retrogradação é calculado a partir da viscosidade final da amostra, diminuindo-se o valor da viscosidade mínima à temperatura constante.

Os valores obtidos para tendência à retrogradação foram 949,0 e 1069,0 cP para as FR e 1654,0 e 1635,5 cP para as FI dos fornecedores A e $B$, respectivamente, mostrando que farinhas que possuem maiores valores de viscosidade mínima à temperatura constante também possuem maiores valores de tendência à retrogradação.

De acordo com o estudo de RAGAEE \& ABDEL-AAL (2006), baixos valores de tendência à retrogradação indicam baixa razão de retrogradação do amido e sinerese. Entretanto, a retrogradação é uma característica atribuída ao amido e, neste estudo, os maiores valores obtidos foram relacionados à amostra de FI. As FR foram as que apresentaram maiores valores de viscosidade mínima à temperatura constante e os menores valores de tendência à retrogradação.

De acordo com resultados obtidos por RAGAEE \& ABDEL-AAL (2006), as propriedades de pasta de mesclas de $15 \%$ de cereais integrais (cevada, milheto, centeio ou sorgo) e $85 \%$ de farinha refinada de trigo (duro ou mole) mostraram efeito marcado no comportamento de farinha de trigo, onde a quebra e a retrogradação tornaram-se mais consistentes nas mesclas. $\mathrm{O}$ aumento significativo desses dois parâmetros também foi observado no presente estudo, para a mistura de $20 \%$ FI com $80 \%$ de FR, a que mais se assemelha a do estudo de RAGAEE \& ABDEL-AAL (2006).

\section{CONCLUSÃO}

Os resultados obtidos neste trabalho permitem concluir, em relação às propriedades de pasta, que a adição de farinha integral à farinha refinada proporciona diminuição dos valores de viscosidade máxima e aumento dos valores de viscosidade final e de tendência à retrogradação, sendo encontrados maiores valores de quebra e de viscosidade mínima à temperatura constante em mesclas com pico de viscosidade elevado, normalmente menos estáveis ao aquecimento. Assim, as características de viscosidade deverão ser observadas de forma conjunta, pois afetarão o processo de produção de pães e o armazenamento. Além disso, os resultados mostraram que o RVA pode ser usado para ajudar a formular mesclas de farinha integral com farinha refinada com determinadas propriedades de pasta.

\section{AGRADECIMENTOS}

Os autores agradecem o suporte financeiro da Coordenação de Aperfeiçoamento de Pessoal e Nível Superior (CAPES), pela concessão da bolsa de doutorado, e ao apoio da Universidade Federal de Santa Catarina, da Embrapa Trigo e da Universidade de Passo Fundo, em especial das funcionárias do Laboratório de Cereais desta última. 


\section{REFERÊNCIAS}

AACC (AMERICAN ASSOCIATION OF CEREAL CHEMISTRY). Internacional approved methods of analysis. 10.ed. St. Paul, MN, 2000. Approved November 8.

ABIP (ASSOCIAÇÃO BRASILEIRA DA INDÚSTRIA DE PANIFICAÇÃOE CONFEITARIA). Perfil do Setor de Panificação no Brasil. Acesso em: 04 out. 2011. Online. Disponível em: <http:// www.abip.org.br/perfil_internas.aspx?cod=35>.

BRABENDER GmbH \& Co. KG. Acesso em: 28 jul. 2011. Online. Disponível em: 〈http://www.brabender.com>.

BRANDOLINI, A. et al. Storage-induced changes in einkorn (Triticum monococcum L.) and breadwheat (Triticum aestivum L. ssp. Aestivum) flours. Journal of Cereal Science, v.51, p.205212, 2010. Disponível em: <http://www.sciencedirect.com/ science/article/pii/S0733521009001805>. Acesso em: 20 set. 2012. doi: 10.1016/j.jcs.2009.11.013.

BRASIL. Ministério da Agricultura, Pecuária e Abastecimento. Resolução Normativa n.8, de 02 de junho de 2005. Regulamento Técnico de Identidade e Qualidade da Farinha de Trigo. Diário Oficial [da] República Federativa do Brasil, Brasília, DF, n.105, p.91, 3 jun. 2005. Seção 1.

BRASIL. Ministério da Agricultura, Pecuária e Abastecimento. Instrução Normativa n.38, de 30 de novembro de 2010. Regulamento técnico do trigo. Diário Oficial da República Federativa do Brasil, Brasília, DF, n.29, p.2, 1 dez. 2010. Seção 1.

CONAB (COMPANHIA NACIONAL DE ABASTECIMENTO). Acompanhamento da safra brasileira de grãos. 12o Levantamento. Brasília, set. 2011. Acesso em: 08 nov. 2011. Online. Disponível em: <http://www.conab.gov.br/OlalaCMS/uploads/ arquivos/11_09_19_09_49_47_boletim_setembro-2011.pdf>.

COPELAND, L. et al. Form and functionality of starch. Food Hydrocolloids, v.23, p.1527-1534, 2009. Disponível em: <http:// www.sciencedirect.com/science/article/pii/S0268005X08002397>. Acesso em: 18 set. 2011. doi: 10.1016/j.foodhyd.2008.09.016.

COSTA, M.G. et al. Qualidade tecnológica de grãos e farinhas de trigo nacionais e importados. Ciência e Tecnologia de Alimentos, v.28, n.1, p.220-225, 2008. Disponível em: <http://www.scielo.br/ pdf/cta/v28n1/30.pdf>. Acesso em: 11 dez. 2012. ISSN 0101-2061.

FIEP (FEDERAÇÃO DAS INDÚSTRIAS DO ESTADO DO PARANÁ). Programa de aumento das vendas dos produtos paranaenses - farinha de trigo, v.1, 2006. Acesso em: 28 set. 2011. Online. Disponível em: <http://www.fiepr.org.br/fiepr/ conselhos/agroindustria_alimentos/uploadAddress/Relat\%C3\% B3 rioFarinhadeTrigo0606.pdf>.

FRANCO, C.M.L et al. Structural and functional characteristics of selected soft wheat starches. Cereal Chemistry, v.79, n.2, p.243248, 2002. Disponível em: <http://cerealchemistry.aaccnet.org/ doi/pdf/10.1094/CCHEM.2002.79.2.243>. Acesso em: 03 out. 2011. doi: 10.1094/CCHEM.2002.79.2.243.

IGC (INTERNATIONAL GRAINS COUNCIL). Grain market report, n.416, Oct. 2011. Acesso em: 08 nov. 2011. Online. Disponível em: <http://www.igc.int/en/downloads/gmrsummary/ gmrsumme.pdf $>$.
JANE, J. et al. Effects of amylopectin branch chain length and amylose content on the gelatinization and pasting properties of starch. Cereal Chemistry, v.76, n.5, p.629-637, 1999. Disponível em: <http:// cerealchemistry.aaccnet.org/doi/pdf/10.1094/CCHEM.1999.76.5.629>. Acesso em: 16 set. 2011. doi: 10.1094/CCHEM.1999.76.5.629.

KOHYAMA, K. et al. A differential thermal analysis of the gelatinization and retrogradation of wheat starches with different amylopectin chain lengths. Carbohydrate Polymers, v.58, p.7177, 2004. Disponível em: <http://www.sciencedirect.com/science/ article/pii/S0144861704002243>. Acesso em: 15 ago. 2011. doi: 10.1016/j.carbpol.2004.06.032.

LEON, E. et al. Pasting properties of transgenic lines of a commercial bread wheat expressing combinations of HMW glutenin subunit genes. Journal of Cereal Science, v.51, p.344349, 2010. Disponível em: <http://www.sciencedirect.com/ science/article/pii/S0733521010000305>. Acesso em: 23 ago. 2011. doi: 10.1016/j.jcs.2010.02.002.

NEWPORT SCIENTIFIC. Instruction manual for the series 4 rapid visco analyzer. Australia: Newport Scientific Pty, 2001. p.1-45. Disponível em: <http://www.newport.com.au/products/ brochures/manuals/RVA-4\%20Operation\%20manual\%2025-0803.pdf >. Online. Acesso em: 10 out. 2011.

PROPAN (PROGRAMA DE APOIO A PANIFICAÇÃO). Programa de desenvolvimento da alimentação, confeitaria e panificação. Acesso em: 10 out. 2011. Online. Disponível em: <http://www.propan.com.br/noticia.php?id=844>.

RAGAEE, S.; ABDEL-AAL, EL-S. Pasting properties of starch and protein in selected cereals and quality of their food products. Food Chemistry, v.95, p.9-18, 2006. Disponível em: <http:// www.sciencedirect.com/science/article/pii/S0308814605000245>. Acesso em: 14 ago. 2011. doi: 10.1016/j.foodchem.2004.12.012.

SAS INSTITUTE CORP. The SAS for Windows, release 6.08. Cary, NC, USA, 1992. 211p.

SHEWRY, P.R. Wheat. Journal of Experimental Botany, v.60, n.6, p.1537-1553, 2009. Disponível em: <http://jxb. oxfordjournals.org/content/60/6/1537.full>. Acesso em: 16 set. 2011. doi: $10.1093 / \mathrm{jxb} / \mathrm{erp} 058$

SINGH, S. et al. Relationship of polymeric proteins with pasting, gel dynamic- and dough empirical-rheology in different Indian wheat varieties. Food Hydrocolloids, v.25, p.19-24, 2011. Disponível em: <http://www.sciencedirect.com/science/article/pii/ S0268005X10000925>. Acesso em: 02 set. 2011. doi: 10.1016/j. foodhyd.2010.05.001.

TESTER, F.; MORRISON, W.R. Swelling and gelatinization of cereal Starches. I. Effects of Amylopectin, amylase, and lipids. Cereal Chemistry, v.67, p.551, 1990. Disponível em: http://www. aaccnet.org/publications/cc/backissues/1990/Documents/67_551. pdf. Acesso em: 12 out. 2011.

WHOLE GRAINS COUNCIL. What is a Whole Grain? Acesso em: 10 out. 2011. Online. Disponível em: <http://www. wholegrainscouncil.org/whole-grains-101/what-is-a-whole-grain>.

ZENG, M. et al. Sources of variation for starch gelatinization, pasting, and gelation properties in wheat. Cereal Chemistry, v.74, n.1, p.63-71, 1997. Disponível em: <http://cerealchemistry. aaccnet.org/doi/abs/10.1094/CCHEM.1997.74.1.63>. Acesso em: 29 set. 2011. doi: 10.1094/CCHEM.1997.74.1.63. 